is thus exactly the same as that of an increased jaw jerk. They described eight patients seen in two centres in the United States with this reflex. It was particularly distinct in seven, four with cerebral and brainstem vascular disease, one with a cerebral tumour, one with a probable cereb 1 llopontine angle tumour, and one with probable encephalomyelitis. The reflex had the advantage when compared with the jaw jerk in that an increased reflex on one side indicated an upper motor neuron lesion predominating on that side. One patient with amyotrophic lateral sclerosis had a weakly positive corneomandibular reflex. Gordon and Bender attribute the first description of this reflex to von Sölder in $1902^{2}$ and detail the various studies of it from that date. It is clearly rare, for they found it to be present in only one of 300 randomly selected neurological patients, a woman with a thoracic myelopathy due to lymphosarcoma and no clinical evidence of brain disease. On the other hand the corneomental reflex was found in $10-15 \%$ of patients, some with no evidence of brain disease.

Neurosurgeons, particularly those dealing with head injuries, are well versed in the examination outlined here of the patient with disturbed consciousness. On the neurological side, as Gordon and Bender's study indicates, that commonest of neurological conditions, cerebrovascular disease, provides the greatest number of patients with dis- turbed consciousness. Understanding of stupor and coma has reached its peak in the U.S.A., ${ }^{3}$ where neurologists in many centres have for many years concerned themselves with patients with "strokes." In the United Kingdom, unfortunately, neurologists as a whole have found themselves unable to look after the large number of patients with cerebrovascular disease. Consequently the level of special skill, and so of teaching, in this branch of medicine has dropped behind that in America.

The time has come to accept the lesson offered by the transatlantic experience, and to consider the creation of regional "stroke units" along the lines of the recently developed coronary care units. These units would need both to care for the patient in the acute phase of his illness and to have full rehabilitation services, which are so hard pressed in this country at present. A considerable expansion of the number of neurologists and specialists in rehabilitation medicine would also be required. Nevertheless, the long-term benefit in the welfare of patients with strokes and in the teaching of neurology demands that we seriously consider the development of stroke units in this country.

\footnotetext{
Gordon, R. M., and Bender, M. B., fournal of Neurology, Neurosurgery and Psychiatry, 1971, 34, 236.

2 Sölder, F. von, Neurologisches Zentralblatt, 1902, 21, 111.

3 Plum, F., and Posner, J. B., The Diagnosis of Stupor and Coma. Oxford, Blackwell, 1966
}

\title{
Detection of Regional Left Ventricular Dysfunction
}

The first 10 years of coronary care have been devoted mainly to the prevention and treatment of dysrhythmias, the common cause of early death after myocardial infarction. Now more attention is paid to conservation of the myocardium, because those patients who are saved from death due to dysrhythmia will eventually succumb to loss of myocardium.

Besides relieving angina, a successful operation of aortocoronary bypass grafting ${ }^{1} 2$ should protect the patient against future infarction not only in the immediate territory of the grafted coronary artery but also in adjacent territories, for collaterals may become available there when atheroma blocks other major coronary vessels. It is now possible to perform revascularization as an urgent operation in patients with preinfarction angina and to consider revascularization combined with excision of the infarct for the critically ill patient who is otherwise unlikely to survive. ${ }^{3}$ Surgical removal of the hindrance to proper contraction of the heart owing to a dead segment of left ventricular wall is only beginning to find a place in the treatment of the acute phase of infarction.

Non-elective surgical excision of a chronic left ventricular aneurysm or of damaged myocardium has been successfully practised for nearly a decade, ${ }^{4}$ but improvements in diagnostic techniques and in knowledge of the effect of aneurysm on total left ventricular function have greatly aided the selection of patients. Consequently more patients are now held to be suitable for aneurysmectomy, and this can often profitably be combined with revascularization of adjacent parts of the heart.

The development of a left ventricular aneurysm or even of an extensive dead area of myocardium after infarction greatly lowers the efficiency of the ventricle. ${ }^{5}$ The increased size of the cavity means that a greater tension is sustained in the myocardial wall for the same peak systolic ejecting pressure. As wall tension is a major determinant of the myocardium's metabolic need, 6 it may render an already critical coronary blood supply even more inadequate, with consequent worsening of heart failure and angina. Failure of an aneurysm to discharge its contents may be followed by thrombosis of blood within it and consequent embolization.

The incidence of ventricular aneurysm at postmortem examination of patients who had had a myocardial infarction has been found to be $3.5 \%,{ }^{7}$ but its precise diagnosis in life has been limited by the need to examine patients by contrast angiocardiography. An aneurysm should be suspected if left ventricular failure persists after myocardial infarction and particularly if it follows a first infarct. An electrocardiogram is usually helpful, because in most cases of aneurysm it shows persistent elevation of the ST segment in leads over the infarct. This is a well-established empirical observation, though its cause is not yet understood. $90 \%$ of aneurysms are anterolateral or apical ${ }^{8}$ and usually result from occlusion of the anterior descending branch of the left coronary artery; 9 less often they protrude solely inferiorly or posteriorly. This common place for aneurysms means that they often show up on the plain radiograph of the chest, but, when the aneurysm is complicated by general left ventricular dilatation, any characteristic local deformation of the left ventricular border is likely to become obscured. Inferior aneurysms may sometimes be seen through the stomach gas bubble if they deform the diaphragm, and the more posterior ones often indent the oseophagus to become visible on a barium swallow. ${ }^{10}$ These are less commonly seen because of the likelihood of concomitant damage to the papillary muscles of the mitral valve, a particularly lethal complication.

To confirm the diagnosis the usual procedure is left ventricular cine angiography. Direct visualization of the contracting cavity in both oblique views enables the existence of an aneurysm to be confirmed or refuted with certainty, and it can be distinguished from generalized myocardial failure. Selective coronary angiography carried out at the same time 
enables bypass grafting to be planned if indicated, preservation of remaining myocardium being vital to the patient's survival.

The common anterolateral or apical aneurysm is usually clearly seen in the right anterior oblique view. Though a neck as such is rare, the point of demarcation between actively contracting wall and immobile aneurysm can be appreciated and the proportion of ventricular wall involved can be calculated.

Direct left ventricular angiography carries a surprisingly small risk even in very sick patients, though there is a slight possibility of dislodging a mural thrombus. Recently the use of a scintiphotographic method for detecting left ventricular aneurysm has been reported by B. L. Zaret and his colleagues from Johns Hopkins Hospital, Baltimore, ${ }^{11} 12$ the source of so many practical applications of nuclear medicine to diagnosis. After intravenous injection of human serum albumin labelled with technetium- $99 \mathrm{~m}$ a scintillation camera records the passage of radioactivity through the heart viewed in the right anterior oblique projection. The size of the left ventricle is then assessed and any regional immobility or systolic bulging can be recognized. A good correlation was obtained with the results of conventional left ventricular cine angiography. Probably over $20 \%$ of the surface of the left ventricle needs to be affected before functional derangement is noticeable, ${ }^{13}$ and the Baltimore technique seemed to enable regional defects of this size to be defined. Though selective coronary angiography still needs to be carried out when surgical resection is contemplated, scintiphotography would enable the large number of people who have had a myocarial infarct to be scanned for the detection of an aneurysm, for the serial estimation of its size, and for postoperative evaluation-all this virtually free from risk, automated, and accurate.

${ }^{1}$ Favaloro, R. G., Journal of Thoracic and Cardiovascular Surgery, 1969, $58,178$.

Johnson, W. D., and Lepley, D., jun., fournal of Thoracic and Cardiovascular Surgery, 1970, 59, 128.

3 Hill, J. D., et al., Circulation, 1971, 43, Supplement No. 1, p. 105.

- Effler, D. B., Westcott, R. N., Groves, L. K., and Scully, N. M., Archives of Surgery, 1963, 87, 249.

${ }^{5}$ Klein, M. D., Herman, M. V., and Gorlin, R., Circulation, 1967, 35, 614 .

Sonnenblick, E. H., Ross, J., jun., and Braunwald, E., American fournal of Cardiology, 1968, 22, 328.

Dubnow, M. H., Burchell, H. B., and Titus, J. L., American Heart Fournal, 1965, 70, 753.

- Gorlin, R., Klein, M. D., and Sullivan, J. M., American fournal of Medicine, 1967, 42, 512.

Abrams, D. L., Edelist, A., Luria, M. H., and Miller, A. J., Circulation, $1963,27,164$

10 Baron, M. G., Circulation, 1971, 43, 762.

11 Zaret, B. L., Strauss, W., Hurley, P. J., Natarajan, T. K., and Pitt, B., New England fournal of Medicine, 1971, 284, 1165

12 Zaret, B. L., Strauss, H. W., Hurley, P. J., and Pitt, B., Circulation, 1970, 42, Supplement No. 3, p. 120.

13 Herman, M. V., and Gorlin, R., American fournal of Cardiology, 1969, 23, 538 .

\section{Early Deaths}

It might at first sight seem illogical, even antisocial, to pay close attention to a few deaths more or less among the youngest human inhabitants of a planet threatened with ecological disaster largely as a consequence of an unprecedented population explosion. But even apart from the ethical and humanitarian considerations there are sound reasons for continuing to regard a community's mortality rates during early childhood as useful indicators of the quality of life in general and the sociomedical services in particular. The fact that the data can be recorded so readily, so soon, and so objectively explains the use of changes in the infant mortality rate to assess the effects of introducing a comprehensive health service into a relatively underprivileged community. ${ }^{1}$ Even where, as in the United Kingdom, the focus of interest has for some time been moving from the sheer numbers of the early deaths to the quality of life offered to the survivors, ${ }^{2}$ it is recognized that the two are related. And the mortality rates combine the attractive advantages of ready availability and objectivity-at least of the fact of death itself.

But the interpretation of favourable changes in mortality as pointing to comparable changes in the quality of the survivors' lives is far from straightforward. An obvious and topical example is provided by children born with certain types of congenital malformation such as myelomeningocele. ${ }^{3-5}$ Indiscriminate lowering of the early mortality may impose terrible burdens on the survivors. But for the overwhelming majority of infants, the normal and healthy, there is hope and increasing evidence that the measures which lower mortality tend to produce a corresponding improvement in the quality of life offered them, at least in our present stage of sociomedical development. For instance, the recent findings of G. Rawlings and colleagues ${ }^{6}$ in surviving infants of very low birth weight are much more encouraging than those reported earlier by C. M. Drillien. ${ }^{7}$ The deaths do in fact appear to represent one end of the "continuum of reproductive casualty" postulated by $\mathbf{A}$. $\mathbf{M}$. Lilienfeld and B. Pasamanick, ${ }^{8}$ at the other end of which are "cerebral palsy and perhaps other related conditions." In appromriate circumstances therefore it is legitimate to interpret a reduction of perinatal and infant mortality rates as showing a sociomedical improvement generally and probably also an improvement in the quality of the survivors' lives. But such an interpretation must largely depend on an effective analysis of the causes of the early deaths and a knowledge of the particular community's sociobiological background.

J. H. Elwood and J. Pemberton, ${ }^{9}$ working in Belfast, have been concerned to explain the relatively high infant mortality rate of 29.4 per thousand among the 25,734 live births in their own community during 1963-5. They compared their statistics with those for the 22,386 live births registered in Birmingham during 1964, with an infant mortality rate 21.4 per thousand. Their conclusion is that the expected excess of births to mothers of high parity in Belfast explains only about $12 \%$ of the difference, and that a higher incidence and lower survival rate of infants at the two extremes of the range of birth weight in Belfast accounted for $40 \%$, leaving nearly $50 \%$ unexplained. This in itself is not surprising, but unfortunately they did not cover stillbirths or give any breakdown of the ages at death within the first year. Consequently it is difficult to estimate the relative importance of biological and social factors ${ }^{10}$ or to compare their findings with those of other recent British surveys which had the same objective of learning by comparing communities.

N. R. Butler and D. G. Bonham ${ }^{11}$ on a national scale, and J. K. Russell and colleagues ${ }^{12}$ and D. V. I. Fairweather and colleagues ${ }^{13}$ in comparing Newcastle upon Tyne with Aberdeen, were concerned with stillbirths and early neonatal deaths. These two surveys, based on data specfically collected for the purpose with the active participation of interested pathologists, showed the potential value (as well as the limitations) of attempting to explain as many of the deaths as possible by reference to an agreed nomenclature of necropsy findings as well as to the usual sociomedical factors in the background. The fact that there is a gross (15-fold) difference between Belfast and Birmingham in 